Supporting Information for:

\title{
Quasi-Two-Dimensional Perovskite Nanosheets Based On Triplet Energy Acceptor Molecule With Pure Green Emission Light
}

\author{
Wenyuan Zhao, ${ }^{\dagger, \hbar}$ Ruiling Zhang, ${ }^{\text {,I }}$ Sijia Wang, ${ }^{\dagger, \hbar}$ Daoyuan Zheng,

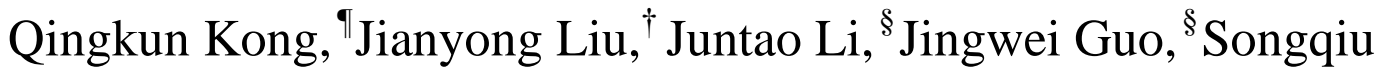 \\ Yang, ${ }^{, \dagger, \$}$ and Keli Han ${ }^{\dagger, *}$
}

\section{AUTHOR ADDRESS}

${ }^{\dagger}$ State Key Laboratory of Molecular Reaction Dynamics, Dalian Institute of Chemical Physics (DICP), Chinese Academy of Sciences (CAS), 457 Zhongshan Road, Dalian, 116023, China.

University of Chinese Academy of Sciences, Beijing, 100049, China.

IIInstitute of Molecular Sciences and Engineering, Shandong University, Qingdao, 266237, China.

${ }^{\S}$ Key Laboratory of Chemical Lasers, Dalian Institute of Chemical Physics (DICP), Chinese Academy of Sciences (CAS), 457 Zhongshan Road, Dalian, 116023, China. 


\section{Content list:}

Figures $\mathrm{S} 1-\mathrm{S} 7$

Fitting parameters of time-resolved PL

Fitting parameters of transient absorption (TA)

Relative PLQY ( $<\mathrm{n}>=2$ NMA NSs as benchmark) 


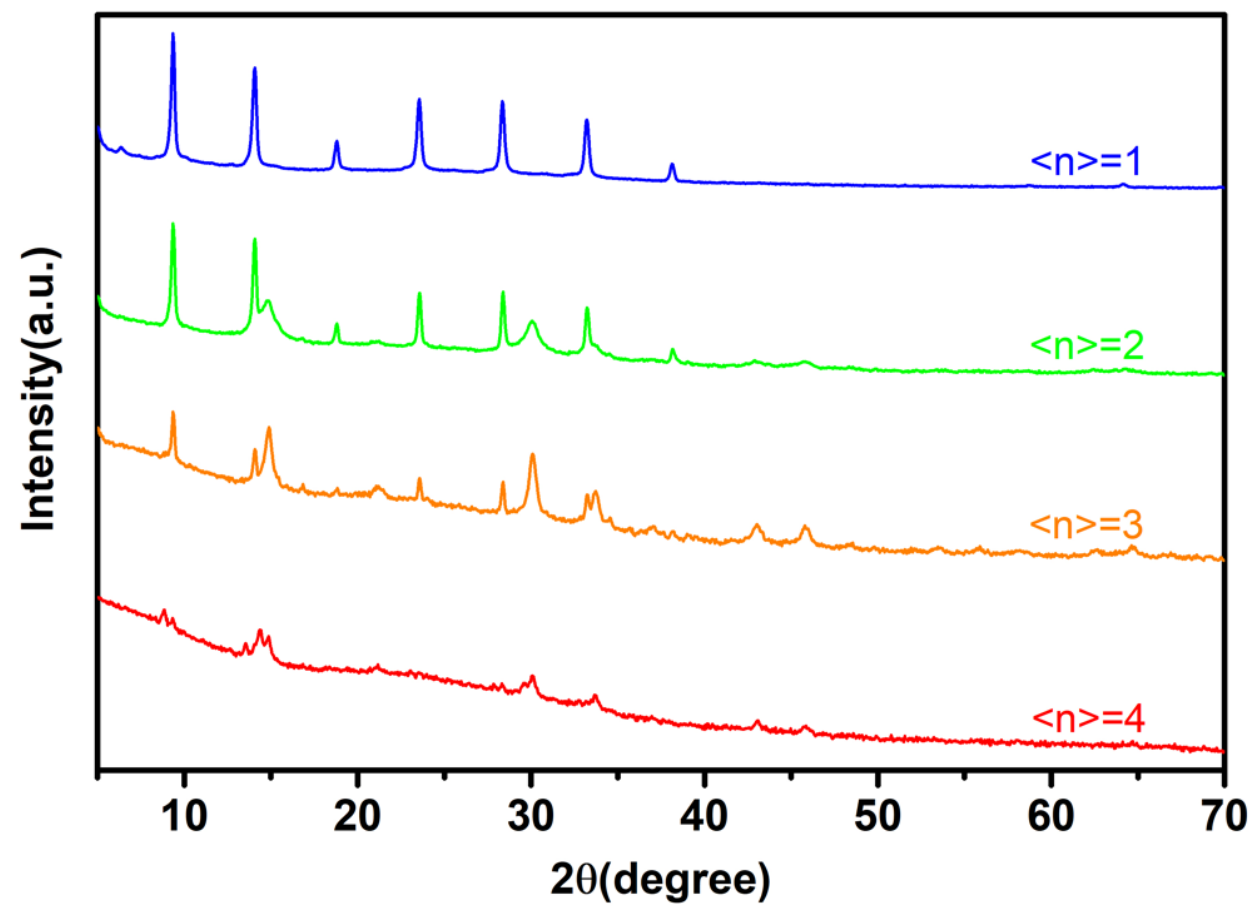

Figure S1. XRD patterns of $(\mathrm{NMA})_{2}(\mathrm{MA})_{<\mathrm{n}>-1} \mathrm{~Pb}_{<\mathrm{n}>} \mathrm{Br}_{3<\mathrm{n}>+1} \mathrm{NS}$ film.

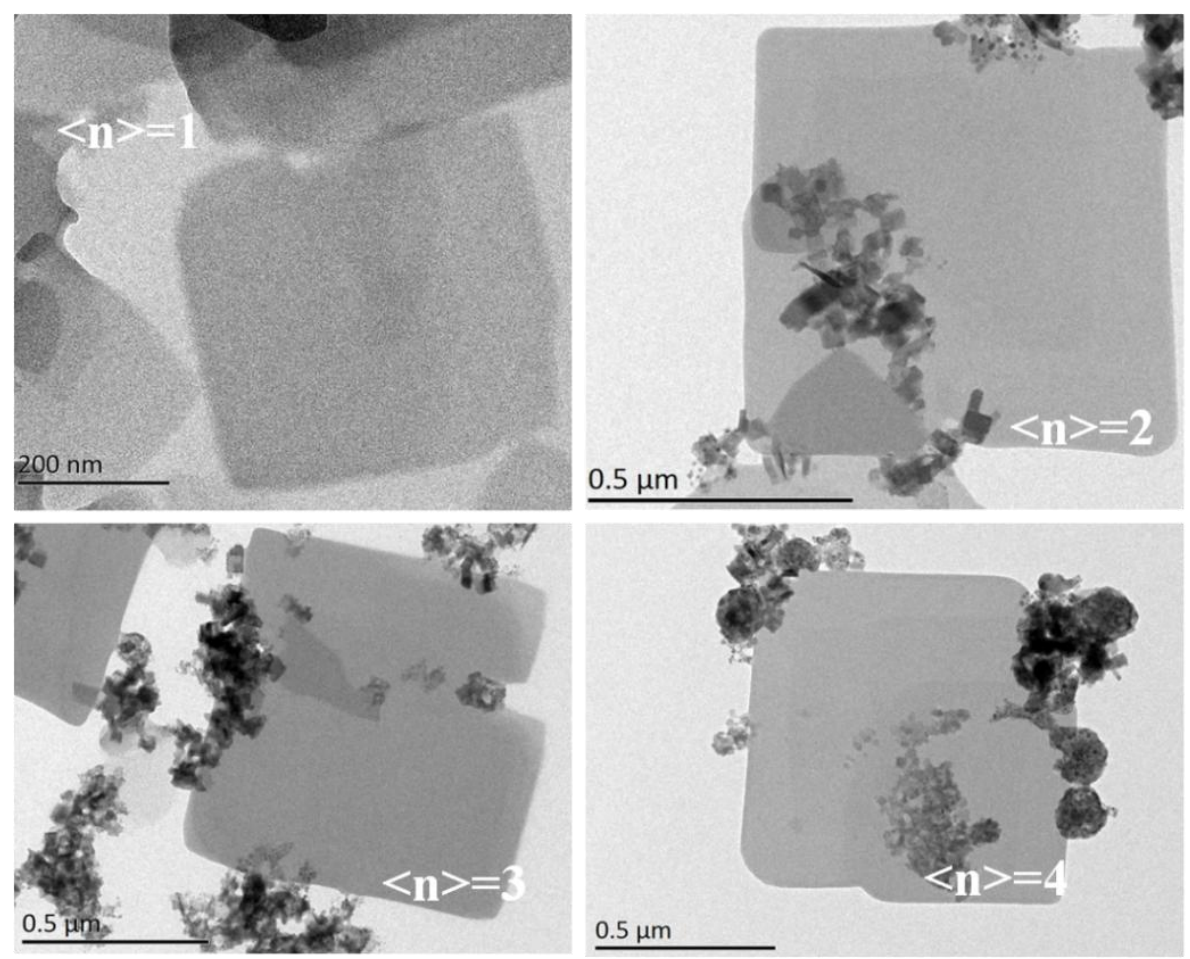

Figure S2. TEM images of $(\mathrm{NMA})_{2}(\mathrm{MA})_{<\mathrm{n}>-1} \mathrm{~Pb}_{<\mathrm{n}>} \mathrm{Br}_{3<\mathrm{n}>+1} \mathrm{NS}$. 


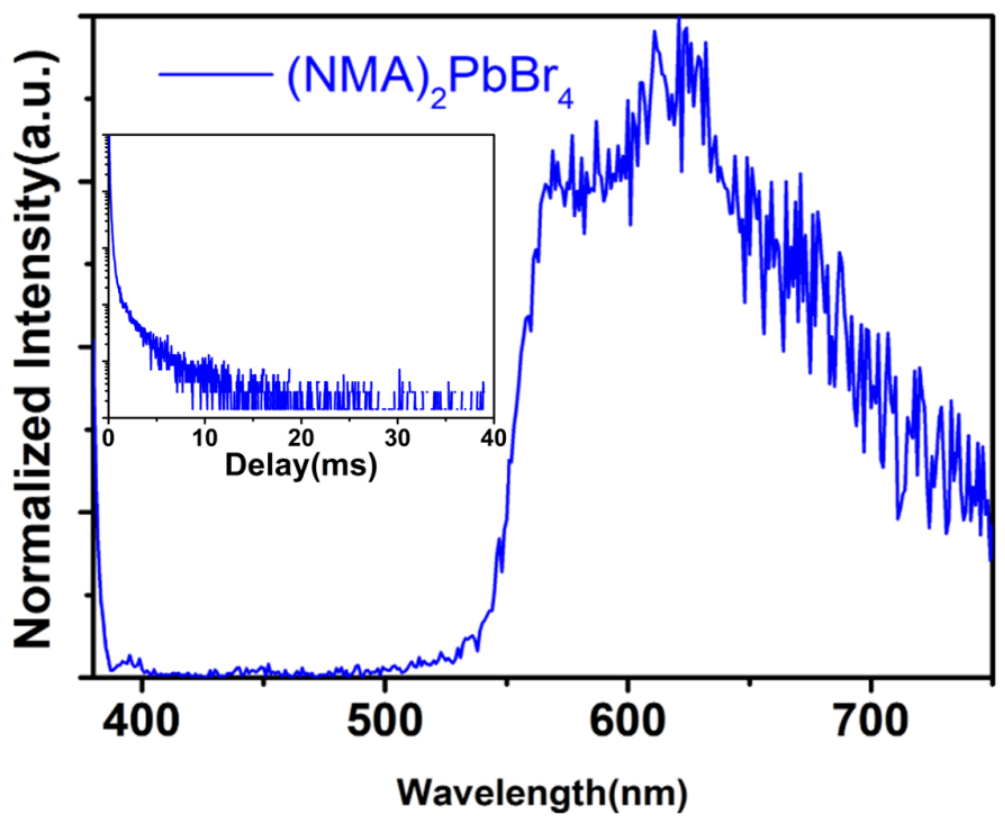

Figure S3. Phosphorescence spectra of (NMA $)_{2} \mathrm{PbBr}_{4} \mathrm{NS}$ with a gae delay time of $0.2 \mathrm{~ms}$. Inset: Time-resolved decay of phosphorescence for (NMA) ${ }_{2} \mathrm{PbBr}_{4} \mathrm{NS}$ at $620 \mathrm{~nm}$.

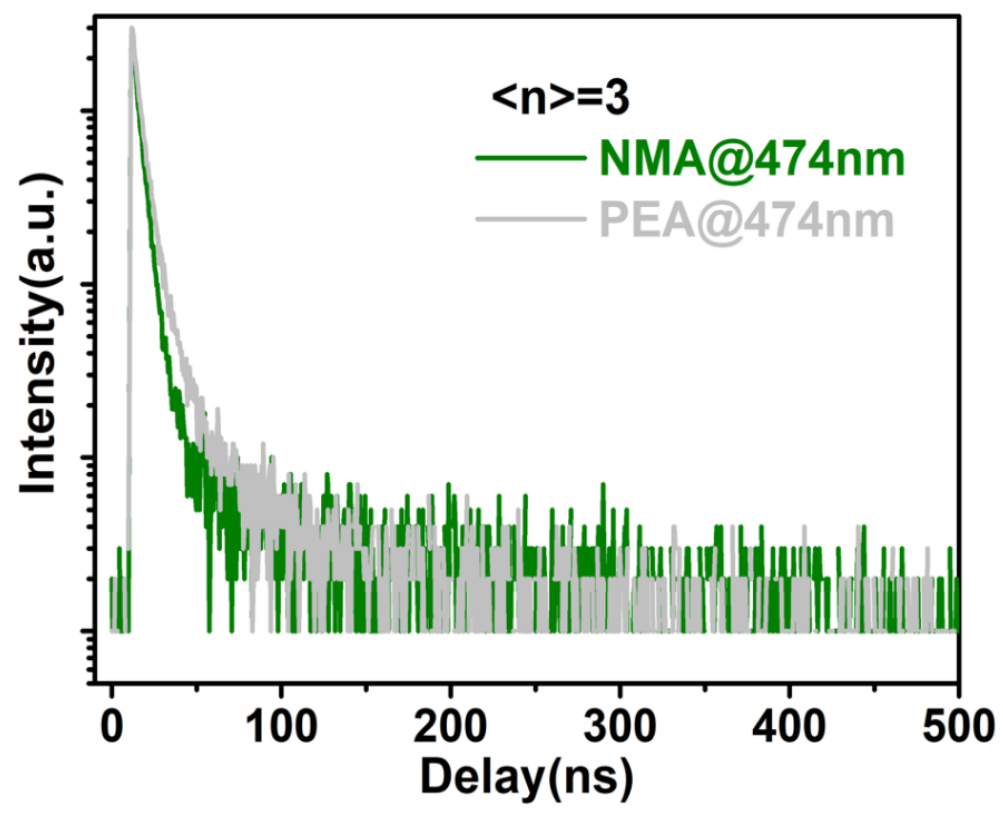

Figure S4. Photoluminescence decay at $\sim 474 \mathrm{~nm}$ of $\langle\mathrm{n}\rangle=3$ NSs. Gray lines represent PEA-based NSs. 

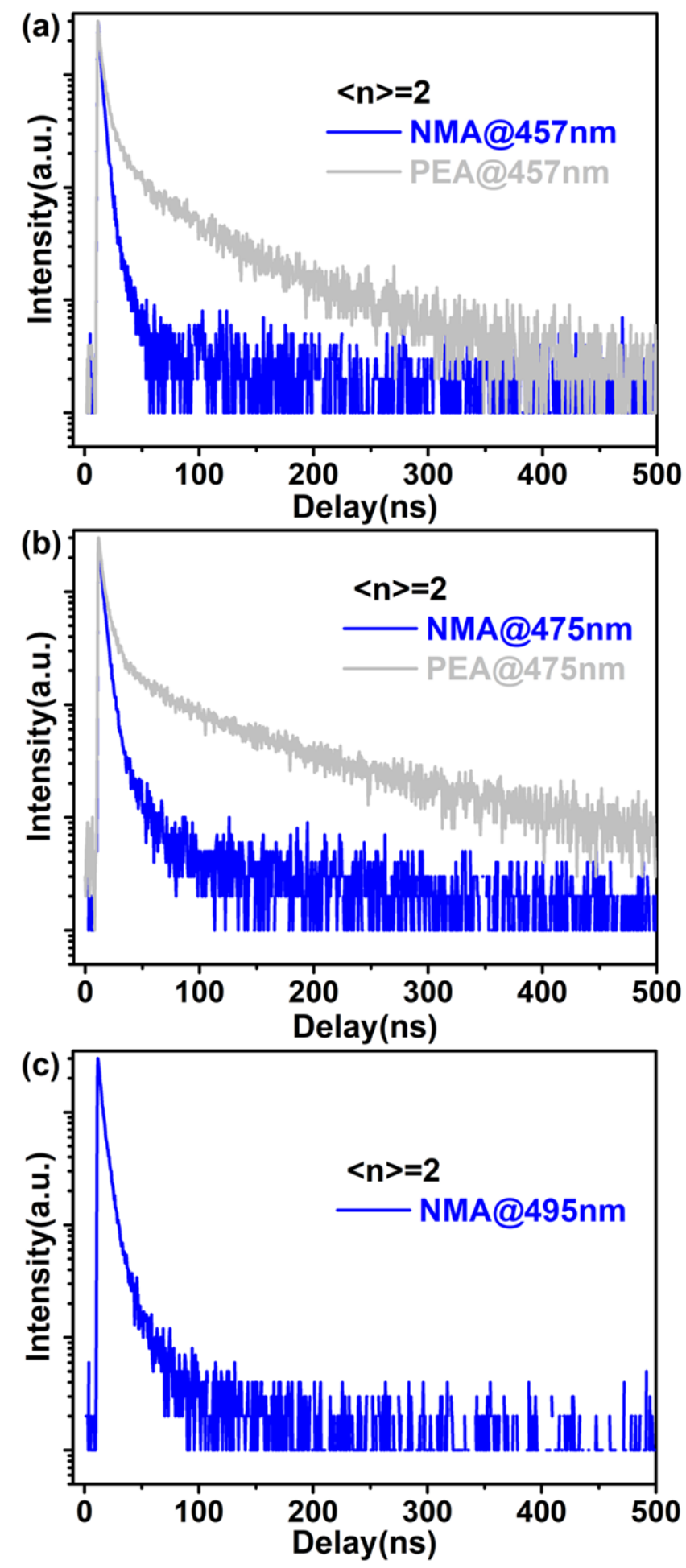

Figure S5. Photoluminescence decay at (a) 457nm, (b) 475nm and (c) 495nm of $<\mathrm{n}\rangle=2$ NSs. Gray lines represent PEA-based NSs. 

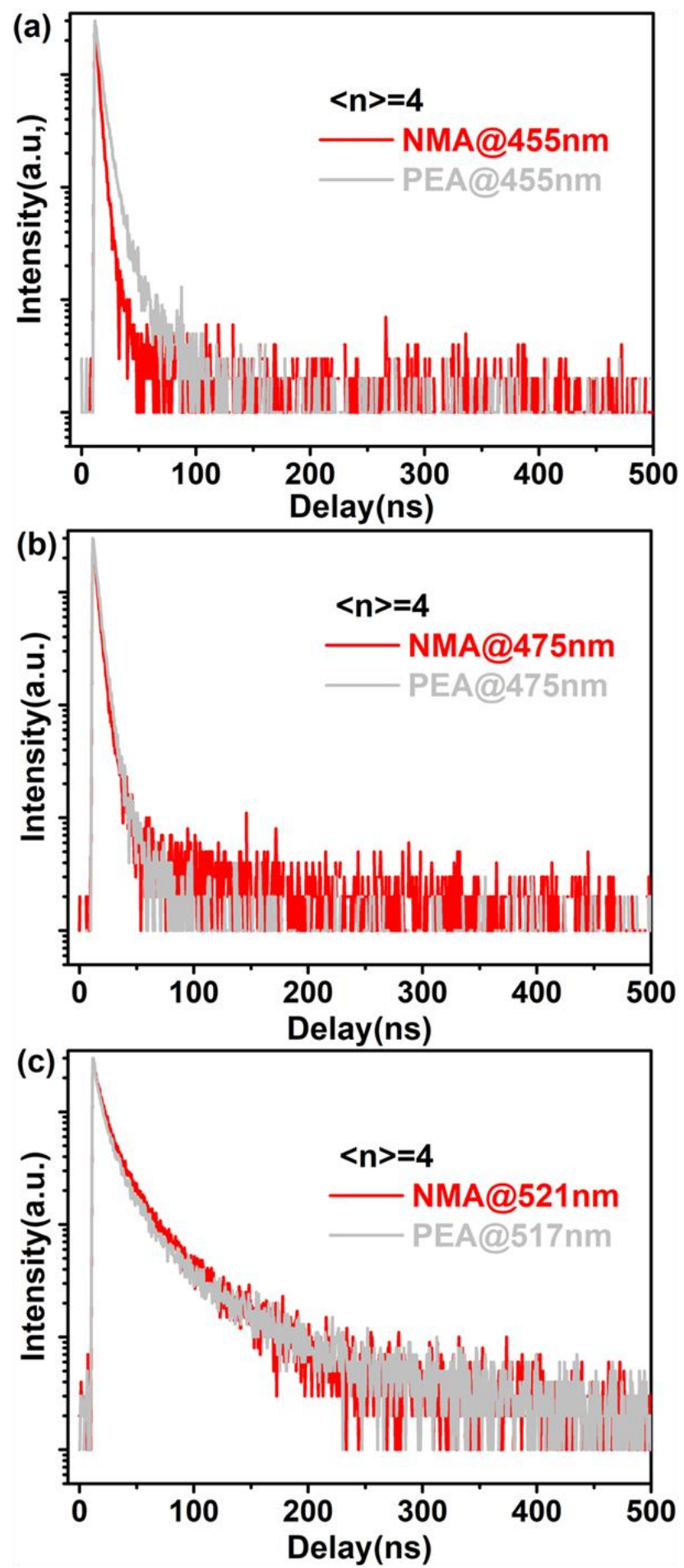

Figure S6. Photoluminescence decay at (a) 455nm, (b) $\sim 475 \mathrm{~nm}$ and (c) $\sim 520 \mathrm{~nm}$ of $<\mathrm{n}>=4$ NSs. Gray lines represent PEA-based NSs. 

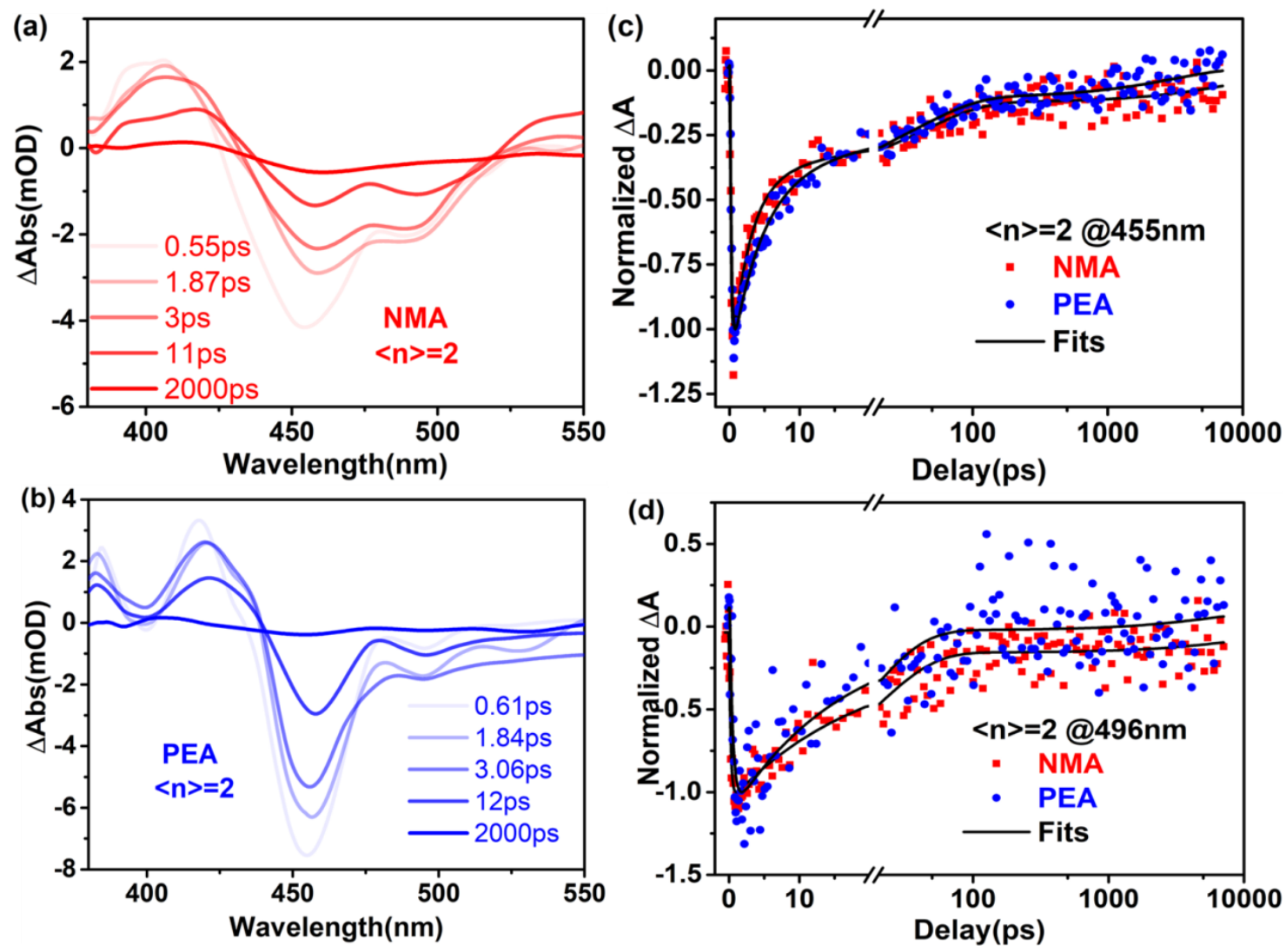

Figure S7. TA spectra of (a) NMA and (b) PEA based $<$ n $>=2$ NSs. Bleach recovery kineticsof $<\mathrm{n}>=3$ NSs at (c) $455 \mathrm{~nm}$ and (d) $496 \mathrm{~nm}$, red squares represent NMA NSs, blue circlesrepresent PEA NSs as references.

\section{Fitting parameters of time-resolved PL}

The PL decay data were fitted with double-exponentials:

$$
\mathrm{PL}(\mathrm{t})=\sum_{i=1}^{2} \mathrm{~A}_{i} e^{-\frac{t}{\tau_{i}}}
$$


Table S1. Fitting parameters of PL for $<n>=2$ NSs

\begin{tabular}{c|c|c|c|c|c|c}
\hline \multicolumn{2}{l|}{} & $\tau_{1}(\mathrm{~ns})$ & $\mathrm{A}_{1}(\%)$ & $\tau_{2}(\mathrm{~ns})$ & $\mathrm{A}_{2}(\%)$ & $\tau_{\text {ave }}(\mathrm{ns})$ \\
\hline & $457 \mathrm{~nm}$ & $2.77 \pm 0.06$ & 95 & $9.42 \pm 0.47$ & 5 & $3.78 \pm 0.12$ \\
$\mathrm{NMA}$ & $475 \mathrm{~nm}$ & $3.84 \pm 0.04$ & 98 & $22.39 \pm 0.99$ & 2 & $5.66 \pm 0.14$ \\
& $495 \mathrm{~nm}$ & $4.01 \pm 0.07$ & 94 & $14.45 \pm 0.53$ & 6 & $5.88 \pm 0.16$ \\
\hline \multirow{2}{*}{ PEA } & $457 \mathrm{~nm}$ & $5.08 \pm 0.06$ & 95 & $63.77 \pm 0.74$ & 5 & $29.54 \pm 0.34$ \\
& $475 \mathrm{~nm}$ & $5.62 \pm 0.06$ & 96 & $89.05 \pm 1.09$ & 4 & $37.75 \pm 0.46$ \\
\hline
\end{tabular}

Table S2. Fitting parameters of PL for $<n>=3$ NSs

\begin{tabular}{c|c|c|c|c|c|c}
\hline \multicolumn{2}{l|}{} & $\tau_{1}(\mathrm{~ns})$ & $\mathrm{A}_{1}(\%)$ & $\tau_{2}(\mathrm{~ns})$ & $\mathrm{A}_{2}(\%)$ & $\tau_{\text {ave }}(\mathrm{ns})$ \\
\hline & $456 \mathrm{~nm}$ & $2.84 \pm 0.06$ & 95 & $8.04 \pm 0.54$ & 5 & $3.55 \pm 0.13$ \\
$\mathrm{NMA}$ & $474 \mathrm{~nm}$ & $3.93 \pm 0.04$ & 98 & $21.22 \pm 1.38$ & 2 & $4.86 \pm 0.11$ \\
& $515 \mathrm{~nm}$ & $6.47 \pm 0.09$ & 90 & $25.83 \pm 0.47$ & 10 & $12.42 \pm 0.21$ \\
\hline & $456 \mathrm{~nm}$ & $5.47 \pm 0.06$ & 97 & $23.49 \pm 0.66$ & 3 & $7.93 \pm 0.15$ \\
& $474 \mathrm{~nm}$ & $4.67 \pm 0.06$ & 97 & $19.87 \pm 0.74$ & 3 & $6.65 \pm 0.14$ \\
& $507 \mathrm{~nm}$ & $6.48 \pm 0.07$ & 94 & $30.8 \pm 0.67$ & 6 & $11.95 \pm 0.21$ \\
\hline
\end{tabular}


Table S3. Fitting parameters of PL for $<\mathrm{n}>=4$ NSs

\begin{tabular}{c|c|c|c|c|c|c}
\hline & & $\tau_{1}(\mathrm{~ns})$ & $\mathrm{A}_{1}(\%)$ & $\tau_{2}(\mathrm{~ns})$ & $\mathrm{A}_{2}(\%)$ & $\tau_{\mathrm{ave}}(\mathrm{ns})$ \\
\hline & $455 \mathrm{~nm}$ & $2.61 \pm 0.08$ & 92 & $6.42 \pm 0.46$ & 8 & $3.30 \pm 0.15$ \\
$\mathrm{NMA}$ & $475 \mathrm{~nm}$ & $3.53 \pm 0.06$ & 96 & $11.42 \pm 0.70$ & 4 & $4.44 \pm 0.14$ \\
& $521 \mathrm{~nm}$ & $9.01 \pm 0.11$ & 90 & $39.94 \pm 0.53$ & 10 & $19.26 \pm 0.25$ \\
\hline & $455 \mathrm{~nm}$ & $4.69 \pm 0.08$ & 93 & $13.74 \pm 0.59$ & 7 & $6.32 \pm 0.18$ \\
& $475 \mathrm{~nm}$ & $4.17 \pm 0.08$ & 94 & $10.67 \pm 0.75$ & 6 & $5.01 \pm 0.17$ \\
& $517 \mathrm{~nm}$ & $8.43 \pm 0.09$ & 93 & $44.16 \pm 0.64$ & 7 & $18.67 \pm 0.25$ \\
\hline
\end{tabular}

Difference of lifetime can be regarded as the character of triplet energy transfer intensity. The influence of energy funnel can be evaluated through quotient of photoluminescence lifetime at $\sim 515 \mathrm{~nm}$ and that at $\sim 456 \mathrm{~nm}$ of PEA-based NSs $\left(\frac{\tau_{\mathrm{PEA}-515 \mathrm{~nm}}}{\tau_{\mathrm{PEA}-456 \mathrm{~nm}}}\right)$ roughly. And the influence of triplet energy transfer can be evaluated through quotient of photoluminescence lifetime at $\sim 456 \mathrm{~nm}$ of PEA-based and NMA-based NSs $\left(\frac{\tau_{\text {PEA-456nm }}}{\tau_{\text {NMA-456nm }}}\right)$ roughly. It can be calculated that the influence of energy funnel for $\langle\mathrm{n}\rangle=3,4$ NSs is 1.51 and 2.95, and that of triplet energy transfer for $\langle\mathrm{n}\rangle=2,3,4$ is $7.81,2.23$ and 1.92, respectively. It can be seen that the more " $<\mathrm{n}\rangle$ ", the more influence of energy funnel and the less triplet energy transfer. But it's abnormal that the lifetime of $\langle\mathrm{n}\rangle=2$ NMA NSs at $495 \mathrm{~nm}$ is much shorter than the lifetime of its reference even at $457 \mathrm{~nm}$, requires further study. 


\section{Fitting parameters of transient absorption (TA)}

The bleach signal at $\sim 450 \mathrm{~nm}$ and $\sim 495 \mathrm{~nm}$ were fitted with triple-exponentials and double-exponentials, respectively:

$$
\begin{aligned}
& \mathrm{XB} @ 450 \mathrm{~nm}(\mathrm{t})=\sum_{i=1}^{3} \mathrm{~A}_{i} e^{-\frac{t}{\tau_{i}}} \\
& \mathrm{XB} @ 495 \mathrm{~nm}(\mathrm{t})=\sum_{i=1}^{2} \mathrm{~A}_{i} e^{-\frac{t}{\tau_{i}}}
\end{aligned}
$$

Table S4. Fitting parameters of XB at 450nm

\begin{tabular}{c|c|c|c|c|c|c|c}
\hline & & $\tau_{1}(\mathrm{ps})$ & $\mathrm{A}_{1}(\%)$ & $\tau_{2}(\mathrm{ps})$ & $\mathrm{A}_{2}(\%)$ & $\tau_{3}(\mathrm{~ns})$ & $\mathrm{A}_{3}(\%)$ \\
\hline$<\mathrm{n}>=2$ & $\mathrm{NMA}$ & $2.67 \pm 0.54$ & 66 & $43.6 \pm 13.3$ & 25 & $>3$ & 9 \\
\hline \multirow{2}{*}{ Pn>=3 } & NMA & $2.40 \pm 0.84$ & 65 & $19.7 \pm 12.3$ & 22 & $>3$ & 9 \\
& & 64 & $40.7 \pm 13.3$ & 27 & $>3$ \\
\hline
\end{tabular}


Table S5. Fitting parameters of XB at $495 \mathrm{~nm}$

\begin{tabular}{c|c|c|c|c|c}
\hline \multicolumn{2}{l|}{} & $\tau_{1}(\mathrm{ps})$ & $\mathrm{A}_{1}(\%)$ & $\tau_{2}(\mathrm{~ns})$ & $\mathrm{A}_{2}(\%)$ \\
\hline$<\mathrm{n}>=2$ & NMA & $19.6 \pm 3.1$ & 88 & $>3$ & 12 \\
& PEA & $15.9 \pm 3.9$ & 91 & $>3$ & 9 \\
\hline$<\mathrm{n}>=3$ & NMA & $15.6 \pm 1.8$ & 87 & $0.61 \pm 0.39$ & 13 \\
& & $12.9 \pm 4.5$ & 77 & & 23 \\
\hline
\end{tabular}

We assume that NMA NS has the similar process with PEA NS. Therefore, the rate of triplet energy transfer, can be calculated with equation below: ${ }^{1}$

$$
\frac{1}{\tau_{T E T}}=\frac{1}{\tau_{N M A, 1}}-\frac{1}{\tau_{P E A, 1}}
$$

Then it can be calculated that $\tau_{\mathrm{TET},<\mathrm{n}>=2}=6.76 \pm 1.54 \mathrm{ps}$, and $\tau_{\mathrm{TET},<\mathrm{n}>=3}=8.8 \pm 5.0 \mathrm{ps}$. 


\section{Relative PLQY (<n>=2 NMA NSs as benchmark)}

Table S6. Relative PLQY for naosheets

\begin{tabular}{c|c|c}
\hline \multicolumn{2}{l|}{} & Relative PLQY $(<\mathrm{n}>=2$ NMA as benchmark $)$ \\
\hline \multirow{2}{*}{ NMA } & $<\mathrm{n}>=2$ & $100 \%$ \\
& $<\mathrm{n}>=3$ & $41 \%$ \\
& $<\mathrm{n}>=4$ & $25 \%$ \\
\hline \multirow{2}{*}{ PEA } & $<\mathrm{n}>=2$ & $50 \%$ \\
& $<\mathrm{n}>=3$ & $66 \%$ \\
& $<\mathrm{n}>=4$ & $60 \%$ \\
\hline
\end{tabular}

\section{References for SI:}

1. C. Mongin, S. Garakyaraghi, N. Razgoniaeva, M. Zamkov and F. N. Castellano, Science, 2016, 351, 369-372. 